\title{
Cryopreserved platelets: from in vitro thrombin generation potential to in vivo safety
}

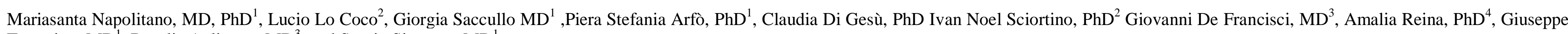
Tarantino, $\mathrm{MD}^{1}$, Rosalia Agliastro, $\mathrm{MD}^{3}$, and Sergio Siragusa, $\mathrm{MD}^{1}$

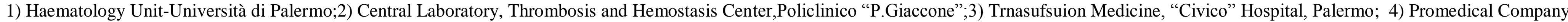

Background: Cryopreservation of platelets (PLTs) at $-80^{\circ} \mathrm{C}$ with dimethyl sulfoxide (DMSO) can extend their shelf life up to 2 years. Cryopreserved PLTs (CRY-PLTs) are reported to have a greater in vivo hemostatic effect than liquid-stored PLTs. Aims of this study were: i.to evaluate the thrombin generation potential of buffy coat derived cryopreserved PLTs (CRY BC-PLT) in comparison with fresh buffy coat derived platelets concentrates; ii.to determine the efficacy and safety of CRY-PLTs transfusion in hematological patients with severe thrombocytopenia.

Results Fourty nine BC-PLTs from 245 healthy volunteer donors (145 males and 100 females, mean age: 48.16.土18.91) were prepared, cryopreserved and analyzed up to 9 months after storage. Cryopreserved PLTs show a good thrombin generation potential that is stably maintained up to 9 months after cryopreservation [ETP (nM min): $529.25 \pm 98.64$ at T0, $558.82 \pm 114.67$ at $\mathrm{T} 3$, at $548.57 \pm 93.38 \mathrm{~T} 6$ and $533.04 \pm 103.15$ at $\mathrm{T} 9$ months, respectively; $\mathrm{PH}(\mathrm{nM}): 132.77 \pm 44.9$ at $\mathrm{T} 0,103.4 \pm 44.9$ at $\mathrm{T} 3$, $108.0 \pm 36.7$ at $\mathrm{T} 6$ and $132.0 \pm 44.6$ at $\mathrm{T} 9$ months ,respectively]. At TGA, fresh BC-PLTs $(n=35)$ had a mean ETP of $760.13 \pm 130.11$,PH was 138.9 \pm 40.2 . Thrombin generation of CRY-BC PLTs is comparable to fresh BC-PLTs, even if slightly decreased. Infusion of CRY-BC PLT (1U) was effective in controlling mucosal bleeding (epistaxis) in two patients with $\mathrm{AL}$ and severe thrombocytopenia. CRY-PLT were also effective when administered for prophylaxis in 3 patients with very low platelets count secondary to chemotherapy. In vivo, thrombin generation is stably maintained up to 24 hours after infusion of 1 Unit of CRY-BC PLTs, without any adverse effect (mean ETP pre-treatment was: $414.13 \pm 160.60$ 24 hours after transfusion : $326.95 \pm 152.54)$.CRY-BC PLTs were safe and they did not determine any thrombotic event.At flow-cytometry, CRY-BC PLTs expressed higher activation markers (CD62P,CD63) than fresh BC PLTs. CRY-BC PLTs are able to significantly decrease the time to clot formation and clot strength, as measured also by thromboelastography. CRY-BC PLTs activation/deterioration is accompanied by an effective

hemostatic in vivo function.

Materials and methods: Buffy coat derived platelets were obtained from 5 buffy coats and pooled. The final PLTs concentrates were leukoreduced by filtration and transferred to a $650 \mathrm{~mL}$ patented cryopreservation kit (Promedical (B) which allowed mixing with DMSO $25 \%$ in a closed system and following removal of supernatant without further manipulations. BC-PLTs were washed prior freezing with removal of at least $84 \%$ supernatant solution, suspended in homologous plasma from 1 of the 5 donors to a final concentration of $200 \mathrm{~mL}$ and frozen at $-80^{\circ}$.CRY- BC PLTs were preserved at $-80^{\circ} \mathrm{C}$ with $6 \% \mathrm{DMSO}$. A system of 3 accessory bags directly connected to the mother bag was adopted for the in vitro study to avoid repeated freezing/thawing of samples at each time point after cryopreservation. In vitro assays were performed before freezing and at 3,6 and 9 months after thawing .Before assay, CRY-BC PLT were thawed at $37^{\circ} \mathrm{C}$ and diluted in plasma to adjust to $300 \times 10^{9} / \mathrm{L}$ PLTs. Fresh BC PLTs underwent the same dilution to adjust to $300 \times 10^{9} / \mathrm{L}$ PLTs. Thrombin generation (TGA) was tested in CRY BC-PLTs and compared to TG potential of fresh BC PLTs. TGA was triggered by the addition of $0.5 \mathrm{pmol} / \mathrm{L}$ of recombinant human tissue factor. Endogenous thrombin potential (ETP) and peak height (PH) were determined. Flow Cytometry assays for PLTs activation markers and thromboelastography were also determined on each sample. CRY-BC PLTs ,separately prepared according to the above described method for in vivo study, were infused in five hematological patients with acute leukemia $(A L)$ and severe thrombocytopenia (PLTs $<10 \times 10^{9} / \mathrm{L}$ ) participating to the trial NCT02032134.CRYBC PLTs were transfused to control epistaxis $(n=2)$ and for prophylaxis $(n=3)$. Patients were observed up to 7 days after infusion and the occurrence of any side effect was registered. An increase in PLTs count was observed only in one case, under prophylaxis, but bleeding was successfully controlled or prevented in all cases. Plasma from patients transfused with CRY-BC PLTs was tested for TGA pre-treatment and 24 hours after treatment.

Conclusions: Cryopreserved PLTs have an enhanced hemostatic activity and a good thrombin generation potential. They are effective and safe in preventing and controlling bleeding, being available in emergency/urgency settings also for patients with acute leukemia and severe thrombocytopenia.

TO

T3

T6

T9

ETP $(\mathrm{nM} \min ) \quad \mathbf{5 2 9 . 2 5} \pm 98.64 \quad \mathbf{5 5 8 . 8 2} \pm \mathbf{1 1 4 . 6 7} \quad \mathbf{5 4 8 . 5 7} \pm 93.38 \quad \mathbf{5 3 3 . 0 4} \pm \mathbf{1 0 3 . 1 5}$

PH (nM)
103.4 \pm 44.9
Thrombin generation parameters of cryopreserved platelets up to 9 months after cryopreservation 